\title{
Chorioretinal coloboma: clinical presentation complications and treatment alternatives
}

\begin{abstract}
Choroio Retinal colobomas rare congenital anomalies developed due to incomplete closure of the embryonic fissure. They present with different clinical conditions depend on extent and effected part of ocular tissues such as macula and optic nerve. The most common complication of Choroio Retinal colobomas is retinal detachment and due to histopathological nature of colobomas the success rate of retinal detachment surgery is not high even with advance vitreoretinal techniques.
\end{abstract}

Keywords: choroid colobomas, retinal colobomas, embryonic fissure, retinal detachment, intercalary membrane

\author{
Volume 9 Issue 4 - 2019
}

\author{
Ayse Gul Kocak Altintas \\ University of Health Sciences, Ulucanlar Eye Hospital,Turkey
}

Correspondence: Ayse Gul Kocak Altintas, University of Health Sciences, Ulucanlar Eye Hospital,Turkey, Tel +905327988488, Email aysegulkaltintas@hotmail.com

Received: July 24, 2019 | Published: August 28, 2019

\section{Introduction}

Choroio Retinal coloboma (CRC) characterized by congenital absence of part of the retinal pigment epithelium and choroid, caused by the defective closure of the embryonic fissure, which normally develops gradually between sixth and seventh weeks of fetal life. Colobomas present various parts of ocular tissue such as iris and /or uvea, lens, optic nerve or eyelid. Since it is embriological pathology CRC may also be associated with other ocular pathologies such as cataract, microphthalmia, anophthalmia. ${ }^{1-4}$

\section{Incidance and transmition}

Ocular colobomas are rare condition occurring in only 0.5 to 2.4 infants per 10.000 live births. The retinochoroidal colobomas present most frequently out of whole colobomas that accounting for $60-70 \%$ and reported to be $0.14 \%$ of the general population. ${ }^{1,5-7} \mathrm{CRC}$ can be sporadic or genetically transmitted as an autosomal recessive, autosomal dominant or X-linked trait..$^{2,5,8}$ Uhumwangho and Jalali ${ }^{2}$ reported parental consanguinity in $28.8 \%$ of the 198 patients in their series. Even it can present more frequently in some families as presended by several reports a specific mutation or genetic pathway was not found yet.

\section{Presentation and histopathology}

The severity of visual disability is highly variable dependent on many factors including size of coloboma, extent of macular or optic nerve involvement, and associated anomalies of the globe such as microphthalmos, microcornea, retrobulbar cysts, nystagmus, that these clinical findings coexisted nearly in $90 \%$ of eyes. ${ }^{2,3,7-9}$ Vincent et al. ${ }^{10}$ reported that the visual acuity is generally poor and $87 \%$ of in eyes with colobomas had a visual acuity worse than $20 / 200$. Uhumwangho and Jalali ${ }^{2}$ observed $29.8 \%$ patient with unilateral involvement while $69.7 \%$ patient with bilateral chorioretinal coloboma. Some of the bilateral colobomas shows asymmetrical involvement due to its histopathological nature in both eyes and presented with different visual acuity level. Histologically in eyes with incomplete closure of the embryonic fissure, the inner layer which will develop into the neurosensory retina grows faster than the outer layer, which will to develop into retina pigment epithelium (RPE). This uncoordinated development cause to laterally displacement and eversion of the RPE, so RPE is absent at the site of the coloboma. Since normal choroidal development is influenced by RPE, the defective development of RPE is leading to absence of choroid in the area of coloboma as well. The neurosensory retina splits into two layers near the margin of the lesion, the inner neuroblastic layer, called intercalary membrane (ICM) which is consist of undifferentiated abnormally thin retina continues in the base of the coloboma whereas the disorganized, outer neuroblastic layer fuses with the RPE. As a result of these maldevelopement, coloboma consists of lacks both choroid and RPE, combined with only rudimentary, ill developed retina. In the base of coloboma retina is gradually thins into the ICM with a few blood vessels over the sclera. Due to histological impaired differentiation and anatomic instability of the retina the risk of rhegmatogenous retinal detachment (RRD) is increased. ${ }^{1,6,7,9-13}$

\section{Clinical findings and classifications}

CRCs present as a prominent depigmente white zone within the fundus which usually located in the inferonasal quadrant that is the latest closure part of embryonic fissure. But they have significantly heterogenous clinical appearance according to extent of involvement and effected part of different anatomical areas such as macula and optic nerve. Several classifications for CRCs have been reported based on anatomic localization and severity. ${ }^{2,9}$ Uhumwangho and $\mathrm{Jalali}^{2}$ reported 7 types of colobomas according to Ida Mann classification.

Type 1 - coloboma extending above the anatomic disc,

Type 2 - coloboma extending up to superior border of disc,

Type 3 - coloboma extending below the lower border of disc,

Type 4-coloboma involving the disc only,

Type 5-coloboma present below the disc with normal retina above and below the coloboma,

Type 6-pigmentation present in the periphery,

Type 7-coloboma involving only the periphery (Figure 1).

Gopal $^{11-13}$ described six types of disc involvement in eyes with coloboma as follows:

Type 1-Optic disc located outside the coloboma and having normal looking, 
Type 2-Optic disc located outside the coloboma and having abnormal, dysplastic disc,

Type 3-Optic disc located outside the cohoroidal coloboma having independent coloboma $\&$ the disc either with a concentric or eccentric lesion,

Type 4-The disc included within the coloboma of the choroid having a nearly normal looking disc,

Type 5-The disc included within the coloboma of the choroid having with coloboma of the disc as well,

Type 6-Having no recognizable disc substance inside a large coloboma of the choroid.

It is reported that the both retinal and choroidal vessels patterns are directly affected by the size and area of the coloboma (Figure 2 ). ${ }^{13}$

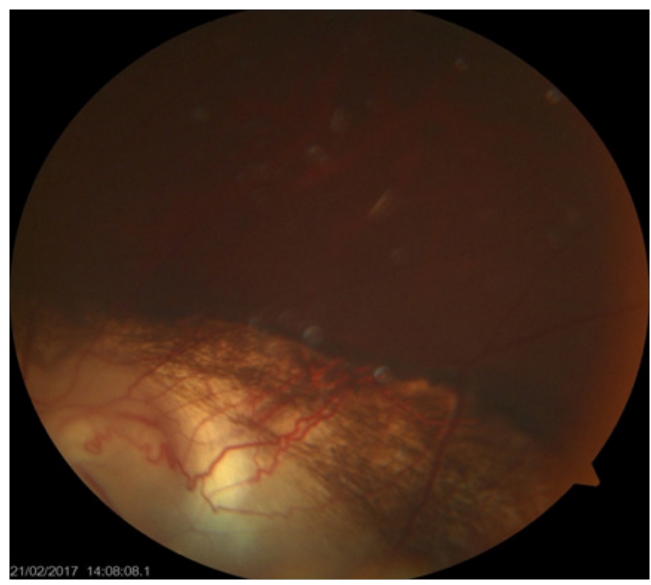

Figure I The course of Retinal blood vessels on choroidal coloboma changed than normal pattern in the lower part of the fundus.

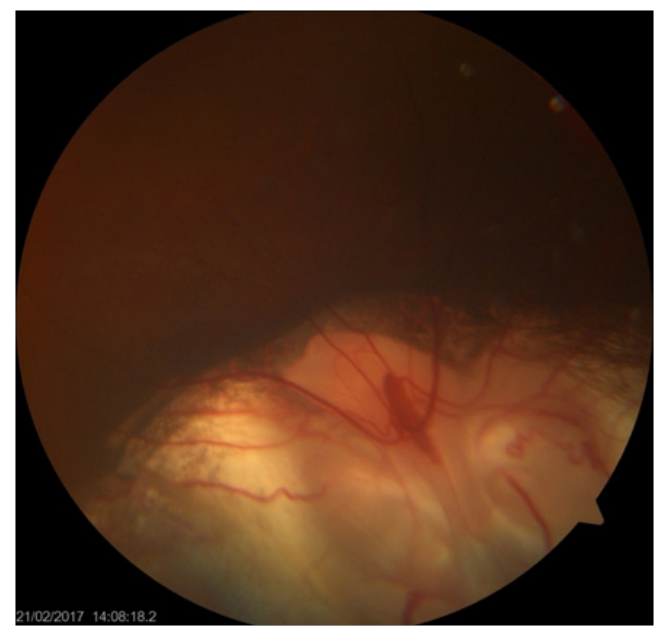

Figure 2 Involvement of optic disc in the coloboma.

\section{Complications}

CRCs can cause different complications due to its histological nature and effected part of ophthalmic tissues. Retinal detachment is the most common complication of CRC and the rate of RRD is significantly higher than the general population. RDD related to CRC have been reported $2 \%$ and up to $8 \%$ in children under age of 10 in whole pediatric RD population group. RRD occurred in up to $40 \%$ of eyes with RCC. ${ }^{1,2,14,15}$ Retinal breaks develop at the junction of the undifferentiated abnormally thin retina and the normal differentiated extra colobomatous retina. Retinal breaks are hard to identify due to absence of retinal pigment epithelium and lack of contrast within these mal developement tissue over the white sclera background. ${ }^{6,9,10,14-16}$ The presence of atrophic holes at the edge of coloboma or hidden breaks in the areas of haemorrhage contribute difficulties in finding the retinal breaks. Therefore exact localization of holes that cause of RRD could not be found in every patient. According to optical coherence tomography (OCT) analysis RRD in eyes with CRC have been found to be most common secondary to a breaks in the abnormally thin inner retina; ICM and communication between both the sub-ICM space and subretinal space. ${ }^{1,26,10,16}$ According to Rishi et al. ${ }^{17}$ report in eyes with optic disc involvement, fluid may enter subretinal space through the colobomatous defect in the optic nerve tissue.

\section{Prophylactic measures}

Prophylactic Laser photocoagulation (PLP) is recommended along the coloboma margin to create a strong chorioretinal adhesion and reinforcing margins, by this way it provides significant benefit for reducing the risk of extracolobomatous retinal detachment. Uhumwangho et al. ${ }^{2}$ reported that retinal detachment occurred in $24.1 \%$ in eyes that did not have PLP comparing to $2.9 \%$ of eyes that received prophylactic photocoagulation. But in colobomas with optic nerve involvement, peripapillary laser photocoagulation on the side papillomacular bundle may cause nerve fiber layer damage, leading to poor visual outcome even it prevents retinal detachment. In addition PLP have some possible other complications including retinal new holes and retinal detachment due to lack of well developed RPE. For preventing these potential complications PLP should be done with light intensity burns that produce a minimal reaction, which is just enough to create the sufficient chorioretinal adhesion. ${ }^{1,2,18,19}$

\section{Management of RRD}

Managing complex RRD in eyes with CRC is remaining a surgical challenge; difficulties may arise due to size of coloboma, locating the retinal breaks and associated ocular anomalies such as microphthalmia, cataract, or lens/iris coloboma. ${ }^{1-3,9,17-19}$ Several surgical techniques have been reported to increase success rate of these eyes. Before development of micro insicional vitreoretinal surgery Scleral buckling had been recommended for several years by different surgeons, such as Wang and Hilton ${ }^{20}$ reported success in buckling the margin of the coloboma with two radial buckles and Patnaik and Kalsi ${ }^{21}$ preferred buckling the entire coloboma. But Scleral buckling had poor success rate in reattachment of detached retina in most of the colobomatous eyes. With current advanced small gauge pars plana vitrectomy techniques with enhanced visualization and illumination systems, the optimal surgical method of choice for managing CRC related RRDs are pars plana vitrectomy, with or without scleral buckle, internal drainage of subretinal fluid and endo-tamponade using long-acting gas (octafluoropropane) or silicone oil. ${ }^{16-18,21-23}$ Rishi et al. ${ }^{17}$ reported a case with successful surgical outcome of pneumatic retinopexy with laser barrage to coloboma margin in a patient with retinal detachment with shallow SRF from a single break in the intercalary membrane with macula sparing retinochoroidal coloboma.

Some other alternative methods have been published such as Hotta $\mathrm{K}$. et al. ${ }^{24}$ recommended cyanoacrylate glue for direct closure of the breaks. But direct closure could not be achieved in most cases because cyanoacrylate is not effective in sealing of detached, extremely thin 
ICM. In contrast, glue may cause progressive atrophy and may enlarge the hole as the ICM contracts. ${ }^{25,26}$ To reduce re-detachment rate and improve the rehabilitation process in long term, silicone oil is also recommended as a long-acting tamponade but it has been reported that silicone oil has the potential risk of getting into the subretinal space through the colobomatous defect. ${ }^{1,18,21-23}$ To prevent this complication the best approach is isolate the coloboma from the rest of the retina and create a border of chorioretinal adhesion. For this purpose internal Laser retinopexy is recommended to be applied at the edge of the coloboma and around the the primary breaks. But it is difficult to create strong adhesion as the choroid and RPE are usually absent around the coloboma similar to PLP. ${ }^{1,20-24}$ Abouammoh et al. ${ }^{24}$ reported that anatomical success was achieved in $87.4 \%$ of their patient and it is significantly higher in eyes that received silicone oil tamponade. According to their results, Cryotherapy is strongly associated with poor anatomical outcomes and placement of an encircling band did not affect final anatomical results. They observed that primary break within the normal retina was found in $60 \%$ of eyes with recurrent RRD. Uhumwangho et al. ${ }^{2}$ observed that $46.7 \%$ patients had a recurrent retinal detachment that required reoperations in their series and visual acuity improved in $56.7 \%$ eyes, unchanged in $30.0 \%$ eyes and worsened in $13.3 \%$ of eyes after several operations in the follow-up period.

\section{Conclusion}

Generally patients with RCC are too young to sufficiently explain the visual changes, so many patients present with longstanding retinal detachments with macular involvement. Vincent et al. ${ }^{10}$ reported that $57 \%$ of patient was under the age of 2 at presentation. Therefore outcomes of retinal detachment surgery in most of cases with RCC have limited benefit even with recent advanced surgical techniques. As a conclusion choroidal coloboma is caused by incomplete closure of the embryonic fissure and congenital absence of the retinal pigment epithelium and choroid in effected part of fundus that appears clinically as a prominent white zone. The surgical management of RRD in eyes with RCC is challenging even with advanced surgical technique and the visual acuity is generally poor.

\section{Acknowledgments}

None.

\section{Conflicts of interest}

Author declares that there is no conflict of interest.

\section{References}

1. Gan NY, Lam WC. Retinal detachments in the pediatric population Taiwan J Ophthalmol. 2018;8(4):222-236.

2. Uhumwangho OM, Jalali S. Chorioretinal coloboma in a paediatric population. Eye. 2014;28(6):728-733.

3. Berk AT, Yaman A, Saatci AO. Ocular and systemic findings associated with optic disc colobomas. J Pediatr Ophthalmol Strabismus. 2003;40(5):272-278

4. Chang L, Blain D, Bertuzzi S, et al. Uveal coloboma: clinical and basic science update. Curr Opin Ophthalmol. 2006;17:447-470.

5. Bermejo E, Martinez-Frias ML. Congenital eye malformations: clinicalepidemiological analysis of 1,124,654 consecutive births in Spain. Am J Med Genet. 1998;75(5):497-504.

6. Daufenbach DR, Ruttum MS, Pulido JS, et al. Chorioretinal colobomas in a pediatric population. Ophthalmology. 1998;105(8):1455-1458.
7. Nakamura KM, Diehl NN, Mohney BG. Incidence ocular findings, and systemic associations of ocular coloboma: a population-based study. Arch Ophthalmol. 2011;129(1):69-74.

8. Gregory-Evans CY, Williams MJ, Halford S, et al. Ocular coloboma: a reassessment in the age of molecular neuroscience. $J$ Med Genet. 2004;41(12):881-891.

9. Hornby SJ, Adolph S, Gilbert CE, et al. Visual acuity in children with coloboma: clinical features and a new phenotypic classification system. Ophthalmology. 2000;107(3):511-520.

10. Vincent D, Venincasa VD, Modi YS, et al. Clinical and Echographic Features of Retinochoroidal and Optic Nerve Colobomas. Invest Ophthalmol Vis Sci. 2015;56(6):3615-3620.

11. Gopal L, Khan B, Jain S, et al. A clinical and optical coherence tomography study of the margins of choroidal. Ophthalmology. 2007;114(3):571-580.

12. Gopal L. A clinical and optical coherence tomography study of choroidal colobomas. Curr Opin Ophthalmol. 2008;19(3):248-254.

13. Gopal L. Pattern of blood vessels in eyes with coloboma. Indian $J$ Ophthalmol. 2013;61(12):743-748.

14. McElnea E, Stephenson K, Gilmore S, et al. Paediatric retinal detachment: aetiology, characteristics and outcomes. Int $J$ Ophthalmol. 2018;11(2):262-266

15. Soliman MM, Macky TA. Pediatric rhegmatogenous retinal detachment. Int Ophthalmol Clin. 2011;51(1):147-171.

16. Schubert HD. Structural organization of choroidal colobomas of young and adult patients and mechanism of retinal detachment. Trans Am Ophthalmol Soc. 2005;103:457-472.

17. Rishi E, Rishi P, Govindarajan MV. Pneumatic retinopexy for the treatment of shallow retinal detachment caused by a retınal break in the intercalary membrane of macula sparing retinochoroidal coloboma. Retin Cases Brief Rep. 2016;10(2):187-190.

18. Wei Y, Li Y, Chen F. Vitrectomy treatment of retinal detachments related to choroidal coloboma involving the disk. Retina. 2014;34(6):1091-1095.

19. Jalali S, Das T. Selection of surgical technique for retinal detachment with coloboma of the choroid. Indian J Ophthalmol. 1994;42(1):27-30.

20. Wang K, Hilton GF. Retinal detachment associated with coloboma of the choroid. Trans Am Ophthalmol Soc. 1985;83:49-62.

21. Patnaik B, Kalsi R. Retinal detachment with coloboma of the choroid. Indian J Ophthalmol.1981;29:345-349.

22. McDonald HR, Lewis H, Brown G, et al. Vitreous surgery for retinal detachment associated with choroidal coloboma. Arch Ophthalmol. 1991;109(10):1399-1402.

23. Pal N, Azad RV, Sharma YR. Long term anatomical and visual outcome of vitreous surgery for retinal detachment with choroidal coloboma. Indian $J$ Ophthalmol. 2006;54(2):85-88.

24. Hotta K, Hirakata A, Hida T. The management of retinal detachments associated with choroidal colobomas by vitrectomy with cyanoacrylate retinopexy. Jpn J Ophthalmol. 1998;42(4):323-326.

25. Gopal L, Badrinath SS, Sharma T, et al. Surgical management of retinal detachments related to coloboma of the choroid. Ophthalmology. 1998;105(5):804-809.

26. Abouammoh MA, Alsulaiman SM, Gupta VS, et al. Surgical outcomes and complications of rhegmatogenous retinal detachment in eyes with chorioretinal coloboma: the results of the KKESH International Collaborative Retina Study Group. Retina. 2017;37(10):1942-1947. 